\title{
Características del Gerente General Relacionadas con Investigación y Desarrollo en Empresas de Chile
}

\author{
Gustavo Barrera Verdugo ${ }^{1^{*}}$, Eugenio Bisama Castillo ${ }^{1}$
}

Resumen: El CEO o gerente general tiene incidencia en las principales decisiones estratégicas y de inversión en las empresas. Investigaciones previas han reconocido condiciones en el gerente general como edad, experiencia, género, participación en la propiedad de la empresa y rango de estudios alcanzados, relacionadas con participación de su organización en investigación y desarrollo. En la presente investigación, se ha utilizado la metodología regresión logística para reconocer si estos patrones son extrapolables a empresas chilenas incluidas en la Encuesta Longitudinal de empresas 2015 del Ministerio de Economía de Chile. Los resultados muestran que la edad, la experiencia, y la condición de único dueño del gerente, se relacionan negativamente con $\mathrm{I}+\mathrm{D}$, y que el rango educacional y el género masculino tienen impacto positivo. Los hallazgos obtenidos son relevantes, pues se reconocen-condiciones que se vinculan con la investigación y desarrollo en empresas en Chile, país que integra el grupo de economías emergentes.

Palabras clave: Investigación y desarrollo; innovación; CEO; gerente general; características gerenciales.

Abstract: CEO's features related to Research and Development in Chilean companies. The CEO or general manager has an impact on the major strategic and investment decisions in business. Previous research recognized features of general manager as age, experience, gender, ownership of the company and level of studies achieved, related with R\&D. In the present investigation, we used logistic regression to recognize whether these patterns are valid in the Chilean companies using as a tool the Business Longitudinal Survey 2015 the Ministry of Economy of Chile. The results show that age, experience, and the manager status as sole owner, impact negatively on R \& D, also, that the educational status and male gender have a positive impact. The findings are relevant because conditions are linked to research and development in companies in Chile, which is part of the group of emerging economies are recognized.

Keywords: Research and development; innovation; CEO; general manager; management characteristics.

Submitted: $\quad$ March $31^{\text {th }} 2016 /$ Approved: $\quad$ October $25^{\text {th }} 2016$

\section{Introducción}

La investigación y desarrollo empresarial apoya a las organizaciones a adaptarse a cambios en su entorno, al apalancar los recursos y capacidades empresariales con su ambiente externo (Ettlie, 1998). También se reconoce que la investigación y desarrollo es fuente primaria de innovación, concepto que a su vez apoya la competitividad y crecimiento de empresas (O’Brien, 2003).

Las principales decisiones de una empresa, entre ellas las actividades de investigación y desarrollo, son determinadas por el chief executive officer (CEO), definido como máxima autoridad operacional de una organización. Las decisiones del CEO determinan la asignación de recursos organizacionales vinculados con investigación y desarrollo (Cazier, 2011; Lin et al., 2011). En Latinoamérica este cargo, tradicionalmente, ha sido denominado gerente general.

En relación con el CEO de una organización, se ha considerado que sus valores y actitudes respecto de la investigación y desarrollo, pueden tener incidencia significativa en las actividades realizadas en este ámbito (Hambrick y Mason 1984), adicionalmente, se ha estimado que aspectos como la edad del CEO, su experiencia, género, nivel educacional y el grado de propiedad que posee sobre la empresa, se vinculan con sus decisiones respecto de investigación y desarrollo en su empresa (Barker y Mueller 2002; Cazier, 2011; Lin et al., 2011).

Esta investigación busca relacionar características del gerente general de empresas chilenas, con participación de su organización en actividades de investigación y desarrollo. En términos específicos, pretende estimar si la participación en propiedad, nivel educacional, edad, años de experiencia profesional y género del gerente general, se vinculan con la ejecución de actividades de investigación y desarrollo en empresas en Chile.

La selección de Chile como país de análisis se pertinente, pues, por más de una década, el gobierno chileno ha promovido la investigación y desarrollo, y ha sido reconocido como poseedor de economía emergente junto a países latinoamericanos como Brasil, México y Colombia (MSCI World Index Stock, 2016). Por tanto, los resultados pueden ser considerados como referente para países latinoamericanos emergentes.

Reconocer relación de las características mencionadas con investigación y desarrollo se considera relevante, pues, bajo una perspectiva teórica, permite estimar si condiciones en los gerentes identificadas en investigaciones previas, explican la participación en actividades de

(1) Universidad Tecnológica de Chile, INACAP. Dirección de Postgrado, Santiago, Chile.

* Autor de correspondencia: gbarrera@inacap.cl 
I+D en empresas chilenas, o si existen diferencias en este país latinoamericano; adicionalmente, desde la perspectiva de la gestión de empresas, ayuda a orientar la selección y formación de gerente generales para facilitar la participación de empresas en I+D; finalmente, desde la perspectiva de políticas públicas, la investigación aporta criterios para la asignación de recursos gubernamentales, al reconocer características del gerente general vinculadas con mayor potencial de I+D.

\section{Marco teórico}

\section{Investigación y desarrollo}

La Organización para la Cooperación y Desarrollo Económicos (2015), indica que investigación y el desarrollo experimental (I+D) "comprende el trabajo creativo llevado a cabo de forma sistemática para incrementar el volumen de conocimientos, incluido el conocimiento del hombre, la cultura y la sociedad y el uso de esos conocimientos para crear nuevas aplicaciones". Respecto de la relevancia de $\mathrm{I}+\mathrm{D}$, durante las últimas dos décadas la literatura académica ha entregado evidencia crucial del rol de estas actividades en el desempeño, generación y sustentabilidad de la ventaja competitiva en una organización (O’Brien, 2003; Hsu, C. W., Lien, Y. C., \& Chen, H., 2015).

En relación con el estudio de variables que afectan la actividad de investigación y desarrollo en una empresa, trabajos previos han examinado diversas variables de incidencia. Se han considerado significativas las características de la industria (e.g. Sujit y Mukherjee, 2005) y la estrategia de diversificación corporativa de las empresas (e.g. Lopez-Sanchez et al., 2006), también, se ha estimado la estructura de propiedad de la empresa como factor que incide en el I + D (e.g. Chen y Hsu, 2009), en último término, las características del CEO han sido reconocidas como variables que afectan las participación de las organizaciones en I + D (e.g. Habrick y Mason 1984; Barker and Mueller, 2002; Cazier, 2011; Lin et al., 2011).

\section{Vínculo de $\mathrm{I}+\mathrm{D}$ con cargos directivos}

El CEO es una función fundamental en la elección de estrategia y nivel de desempeño de la empresa (Minnick y Noga, 2010; Ting, 2013), sus decisiones son relevantes para implementar investigación y desarrollo que facilita la innovación (Chen et al., 2016). Se ha estimado una conexión entre decisiones de administración de los gerentes y sus características (Hambrick y Mason, 1984), y evidenciado que ciertas características demográficas de los gerentes como edad (Barker and Mueller, 2002), educación (Lin et al., 2011) y experiencia (Kor, 2006), inciden en decisiones vinculadas con investigación y desarrollo en las empresas.

En relación con la edad del CEO, Hambrick y Mason (1984) encontraron evidencia de que los CEOs de mayor edad tienden a ser más conservadores, pues presentan mayor conformidad con su posición y seguridad. Del mismo modo, Marshall et al. (2006) sugieren que los gerentes con mayor edad se vuelven más confiados en sus propios recursos de información para tomar decisiones, más conservadores y menos propensos a tomar riesgos. También se ha mostrado, que CEOs más cercanos a la edad de retiro exhiben mayor aversión al riesgo (Matta y Beamish, 2008; Serfling, 2014)
En concordancia con estos estudios, se estima que la edad CEO tendería a impactar negativamente en la participación de la organización en actividades de investigación y desarrollo en Chile.

Respecto de la incidencia de la propiedad del CEO sobre la empresa, Jensen (1993) plantea que la inversión en investigación y desarrollo puede no ser objetivo primario, pues los CEOs prefieren ganancias de corto plazo en vez de incrementar el valor de largo plazo de la empresa a través de investigación y desarrollo. De igual forma, Hirshleifer y Thakor, (1992) afirman que los pagos inciertos provocados por proyectos de investigación y desarrollo, incentivan a los gerentes a evitar participar en ellos. Adicionalmente, se ha reconocido que las empresas familiares presentan menor intensidad de participación en I + D por su mayor aversión al riesgo (Block, 2012; Massis, Frattini, y Lichtenthaler, 2013).

Por el contrario, Barker y Mueller (2002), afirman que la alta participación de los CEOs sobre la propiedad de la empresa, permitiría alinear los intereses de estos con los accionistas, por ello, tendrían mayor incentivo de participar en I + D para aportar al crecimiento de la empresa en el largo plazo.

En general, el efecto esperado de la concentración de propiedad y el gasto en investigación y desarrollo es ambiguo, lo que ha sido confirmado en estudios han concluido la existencia de relación diversa entre la concentración de propiedad del CEO y el gasto en investigación y desarrollo (Ghosh et al., 2007).

A pesar de que la evidencia previa no es concluyente, se estima que la propiedad del CEO tendería a impactar negativamente la participación de la organización en actividades de investigación y desarrollo en Chile, pues la mayoría de empresas en Chile son pequeñas y medianas empresas con vínculo familiar, por tanto, la conducta de sus gerentes debe ser similar a la presentada por directivos de empresas familiares.

En relación con la formación del CEO, Wu, Levitas, y Priem (2005), señalan que existe relación positiva entre capacidad para procesar información, complejidad cognitiva y capacidad para gestionar actividades de investigación y desarrollo, también, Barker y Mueller, (2002) reconocen que el nivel educacional es factor relevante en la orientación cognitiva del CEO, Farag, H., y Mallin, C. (2016) indican que los CEOs postgraduados presentan mayor propensión hacia decisiones riesgosas, adicionalmente, Lee, W. S., \& Moon, J. (2016) estiman relación positiva entre educación del CEO y decisiones riesgosas en la industria aérea, y Lin el al. (2011) evidencian relación positiva entre nivel educacional del CEO gasto en I + D.

De acuerdo con lo planteado, se estima que la formación CEO tendería a impactar positivamente la participación de la organización en actividades de investigación y desarrollo en Chile.

Finalmente, investigaciones previas estiman que el género del CEO puede afectar las decisiones financieras de la empresa. Barua et al. (2010) indican que las empresas que incorporan mujeres en la alta dirección, presentan decisiones financieras más conservadoras, Huang 
y Kisgen (2013) encuentran evidencia que las mujeres CEOs son menos propensas a realizar adquisiciones de empresas y tomar deudas, también, y Faccio et al. (2016) proponen que las mujeres CEOs toman menores riesgos en financiamiento de inversiones. En forma complementaria, se ha reconocido que la investigación y desarrollo es una actividad riesgosa, asociado con retornos inciertos y tardíos (Bromiley, P., Rau, D., y Zhang, Y., 2016), por tanto, las empresas con gerentes de género femenino, debiesen presentar menor propensión a efectuar investigación y desarrollo.

En coherencia con lo anterior, se estima que el género femenino del CEO tendería a impactar negativamente la participación de la organización en actividades de investigación y desarrollo en Chile.

\section{Método}

\section{Muestra}

Se ha utilizado como marco muestral, la tabulación de resultados de Tercera Encuesta Longitudinal de Empresas (ELE3), realizada por el Ministerio de Economía, Fomento y Turismo de Chile (2015). La unidad de análisis son empresas formalizadas con operaciones en Chile.

La Encuesta Longitudinal de Empresas 2015, incluye 7.267 empresas, clasificadas en 12 industrias en forma estratificada, es decir, representa la proporción, según su tamaño y actividad. Para la investigación han sido seleccionadas 3.560 encuestas, considerando tres industrias por su relevancias en el PIB de Chile, estás son Industrias Manufactureras; Comercio al por mayor y al por menor; reparación de vehículos automotores, motocicletas, efectos personales y enseres domésticos; y Actividades Inmobiliarias, Empresariales y de Alquiler. Estás 3 industrias se analizan en 2 tamaños diferentes, que son microempresas, y pyme y grandes empresas. La clasificación de tamaño de empresas, es efectuada por monto de facturación de la empresa, según clasificación de Servicios de Impuesto Internos de Chile.

Esta organización de empresas busca controlar la incidencia del tipo de industria y el tamaño de la organización sobre las variables de estudio. Las pymes y grandes empresas son reunidas en 1 categoría, para conformar un número suficientemente numeroso en la muestra. El gráfico 1, muestra la distribución de empresas incorporadas en el análisis.

Gráfico 1. Distribución de empresas por tamaño en industria

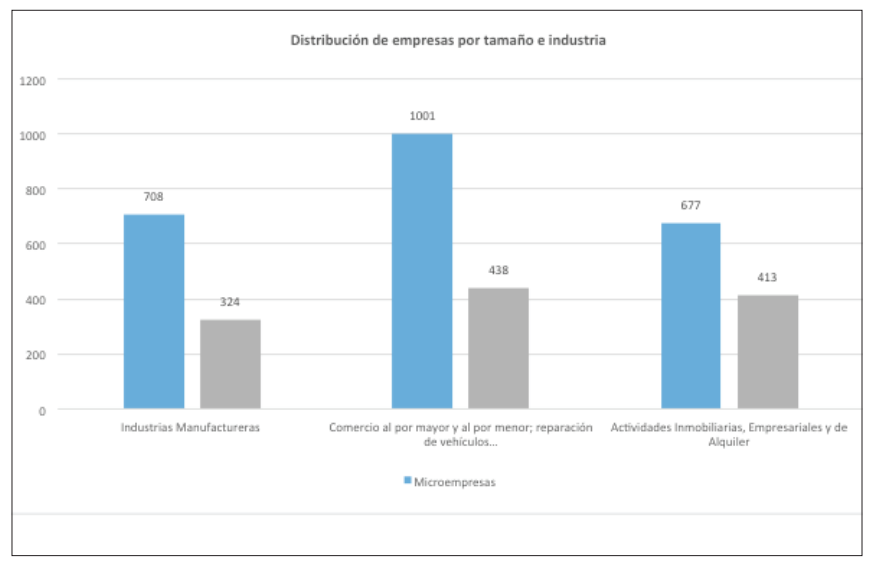

\section{Diseño y procedimiento}

Con el fin de estudiar la participación de una empresa en actividades de investigación y desarrollo, fue seleccionada la pregunta número 1 del módulo F de la encuesta, que consulta “ ¿Su empresa ha realizado trabajos de desarrollo experimental o tecnológico con el objetivo de introducir nuevos productos, procesos, dispositivos, materiales, incluyendo la prueba de prototipo e instalaciones experimentales?", esta pregunta tiene respuesta dicotómica (sí, no).

A través de regresión logística, se estima la probabilidad de que la empresa realice trabajos de desarrollo experimental o tecnológico, es decir, obtener "sí" como respuesta a la pregunta 1 del módulo F, en función de las características del gerente general de la empresa.

El perfil del gerente general fue diferenciado por género, edad, años de experiencia laboral, nivel de propiedad en la empresa que administra y nivel de estudios alcanzados. Para evaluar la formación profesional del gerente general, son considerados los niveles universitaria completa, magíster o doctorado incompleto y magíster o doctorado completo, los encuestados responden sólo una categoría. Las variables independientes son mayoritariamente categóricas de tipo Dummy, sólo la edad y la experiencia del gerente (expresada en años) corresponden a variables escalares. En la siguiente tabla son presentadas las variables incorporadas en análisis de regresión.

Tabla 1. descripción de variables incluidas en modelos de regresión

\begin{tabular}{l|l}
\hline \multicolumn{1}{c|}{ Dependiente } & \multicolumn{1}{c}{ Independientes } \\
\hline $\begin{array}{l}\text { La empresa realiza } \\
\text { actividades de investigación } \\
\text { y desarrollo }\end{array}$ & Edad de gerente general \\
\hline & Años de experiencia del gerente general \\
\hline & Género del gerente general \\
\hline & Gerente general es único dueño \\
\hline & Gerente general es socio o accionista \\
\hline & $\begin{array}{l}\text { Gerente general ha alcanzado estudios uni- } \\
\text { versitarios completos }\end{array}$ \\
\hline & $\begin{array}{l}\text { Gerente general ha alcanzado magíster o } \\
\text { doctorado incompleto }\end{array}$ \\
\hline $\begin{array}{l}\text { Gerente general ha alcanzado doctorado o } \\
\text { magíster completo }\end{array}$ \\
\hline
\end{tabular}

La metodología de análisis utilizada es regresión logística a través de presentación de resultados en 2 modelos. En Modelo 1 propone una regresión global incluyendo las 3.560 empresas, y regresiones por tamaño de empresas, incluyendo microempresa y Pymes y Grandes empresas. En el Modelo 2 se desarrollan regresiones por tamaño de empresas y tipo de industria. 


\section{Resultados}

Descripción de frecuencias porcentuales

El análisis descriptivo de variables estudia frecuencias porcentuales, media aritmética y desviación estándar. La tabla 2, muestra que 18,3\% de las empresas encuestadas declaran realizar trabajos de desarrollo experimental o tecnológico, el 13,5\% de los gerentes son únicos dueños de la empresa y el 39,5\% de los gerentes generales indicar tener propiedad sobre la empresa.

Tabla 2. Frecuentas porcentuales de variables

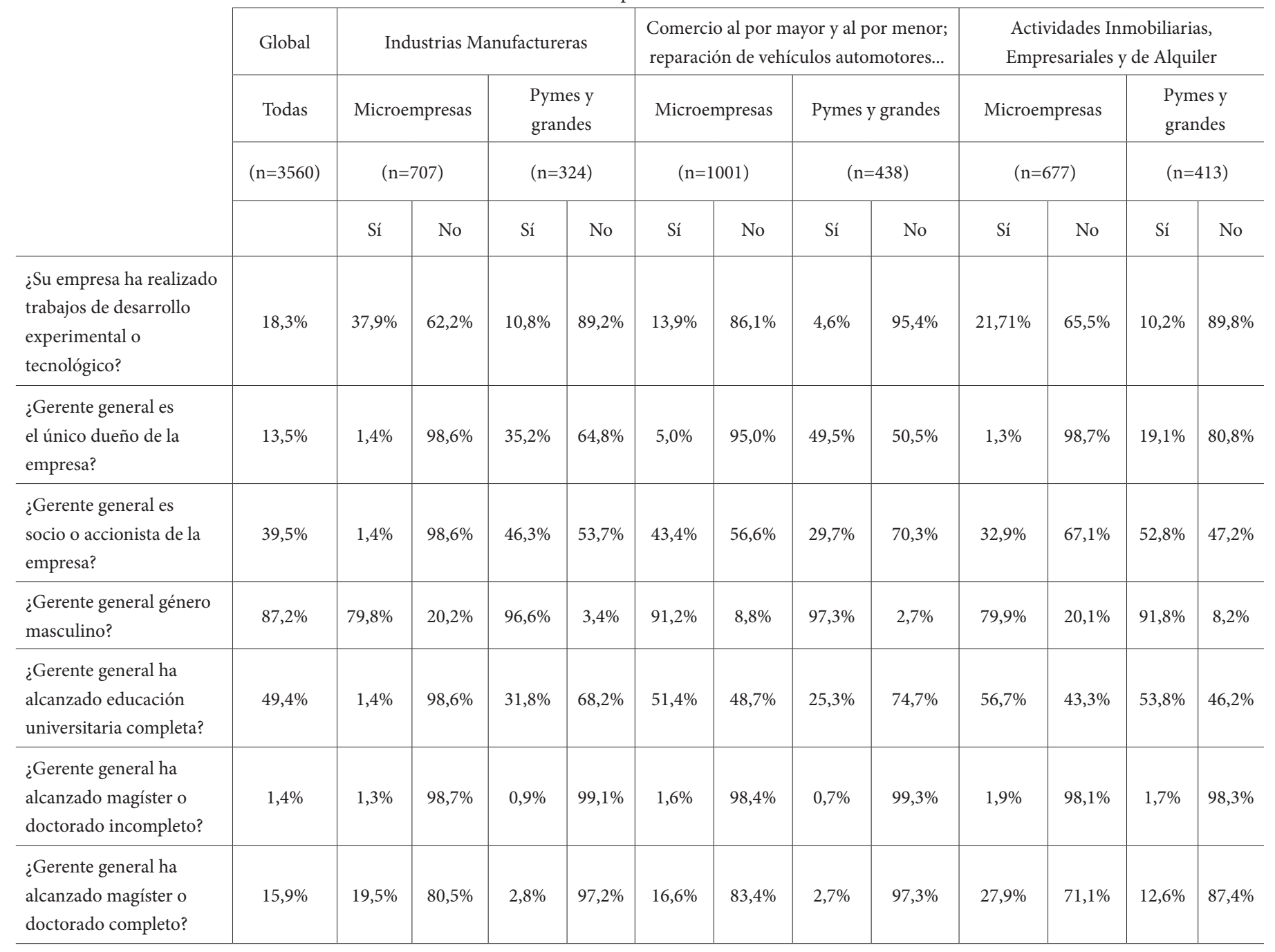

Respecto del nivel educacional de los gerentes generales, el $49,4 \%$ ha alcanzado educación universitaria completa, un $1,4 \%$ responde disponer de estudios de magíster o doctorado incompletos, y un $15,9 \%$ ha finalizado educación de magíster o doctorado.

En relación con el tamaño e industria de la empresa, las microempresas en industria manufacturera presentan mayor participación en actividades de investigación y desarrollo (37,9\%), baja participación de los gerente generales como únicos dueños $(1,4 \%)$, y menor porcentaje de estudios universitarios y de magíster y doctorado que en grandes empresas. La industria con mayor participación de gerentes como únicos dueños o accionistas es Comercio al por mayor y al por menor; reparación de vehículos automotores, motocicletas, efectos personales y enseres domésticos, esta industria también presenta el menor porcentaje de participación en actividades $\mathrm{I}+\mathrm{D}$ para microempresas $(13,9 \%)$, y pymes y grandes empresas (4,6\%). La descripción de muestra presenta que las pymes en general participan en mayor medida en actividades I+D.

En relación con la edad y experiencia del gerente general, se aprecia que la edad promedio del gerente general es menor en microempresas, considerando todas las industrias, también, que la edad promedio del gerente general es 51,7 años y la desviación estándar 10,7 años. La Tabla 3, presenta resultados de media y desviación estándar de edad del gerente general por tamaño de empresa e industria. 
Tabla 3. promedio de edad y experiencia de gerente general

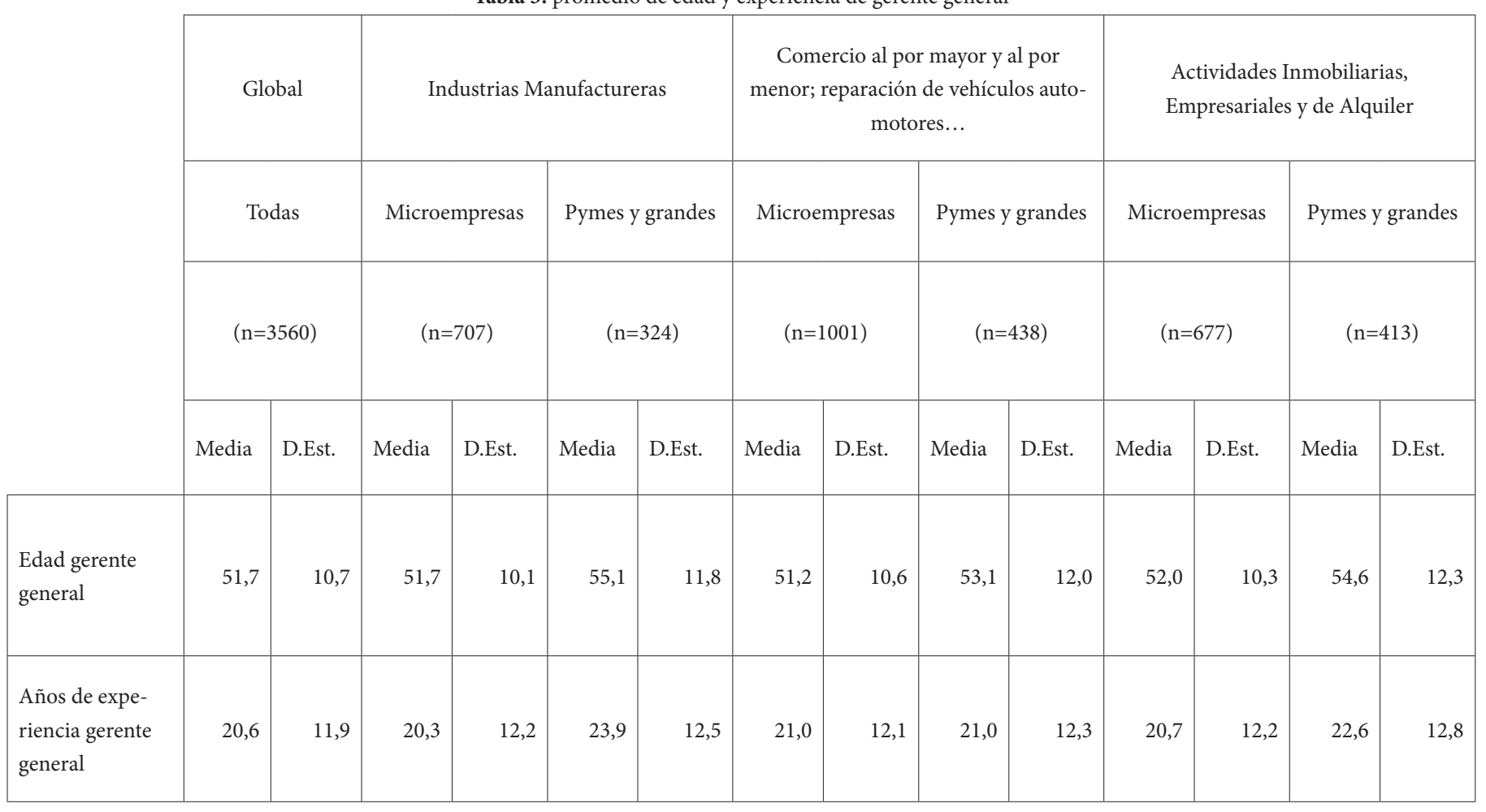

\section{Análisis de correlación}

Al desarrollar matriz de correlación de variables estudiadas, se obtiene que la mayor correlación positiva con variable dependiente se presenta con la variable "alcanzar estudios de magíster o doctorados completos" con correlación 0,124 , y la mayor correlación negativa de variable dependiente se presenta con la variable "gerente general es único dueño de la empresa” con correlación -0,123.

También se encuentra alta correlación entre la edad y los años de experiencia del gerente general. Esta relación es predecible, dado que los ejecutivos con mayor edad tienden a disponer de un mayor número de años de experiencia. Al calcular Alpha de Cronbach para comprobar la consistencia de medición de estas variables se obtiene valor 0,81 , este resultado evidencia que ambas variables podrían ser definidas bajo un concepto.

\section{Modelo 1}

El Modelo 1 de regresión logística presentado en Tabla 4 muestra que, al considerar las 3560 encuestas, el género masculino del gerente general incrementa la probabilidad de investigación y desarrollo en la empresa, también, que las empresas con gerentes generales como únicos dueños tienen menor probabilidad de realizar actividades de investigación y desarrollo. Los valores $\mathrm{P}>/ \mathrm{Z} /$ son 0.000 , lo que valida los Odds Ratio de estas variables con $99 \%$ de confianza.

La educación universitaria completa y de magíster y doctorado completa e incompleta, también obtienen Odds Ratio mayor a 1 y valores valores $\mathrm{P}>/ \mathrm{Z} /$ significativos, esto indica que a mayor estudio del gerentes general, aumenta la probabilidad de que la empresa realice actividades de investigación y desarrollo. El coeficiente Odd Ratio mayor se vincula con el logro de estudios magíster o doctorado completo por parte del gerente, expresado en un valor 3,018. A transformar este valor a probabilidad a través de la formula Probabilidad $=(($ Odds Ratio/ $($ Odds Ratio +1$)$ ), se obtiene que la probabilidad que una empresa con gerente general con estudios de magíster o doctorado completos realice investigación y desarrollo es $75,11 \%$ mayor frente a una empresa sin esta condición. La Tabla 5 presenta probabilidades incrementales de coeficientes significativos al considerar la muestra global. 
Tabla 4. Coeficientes de regresión Modelo 1

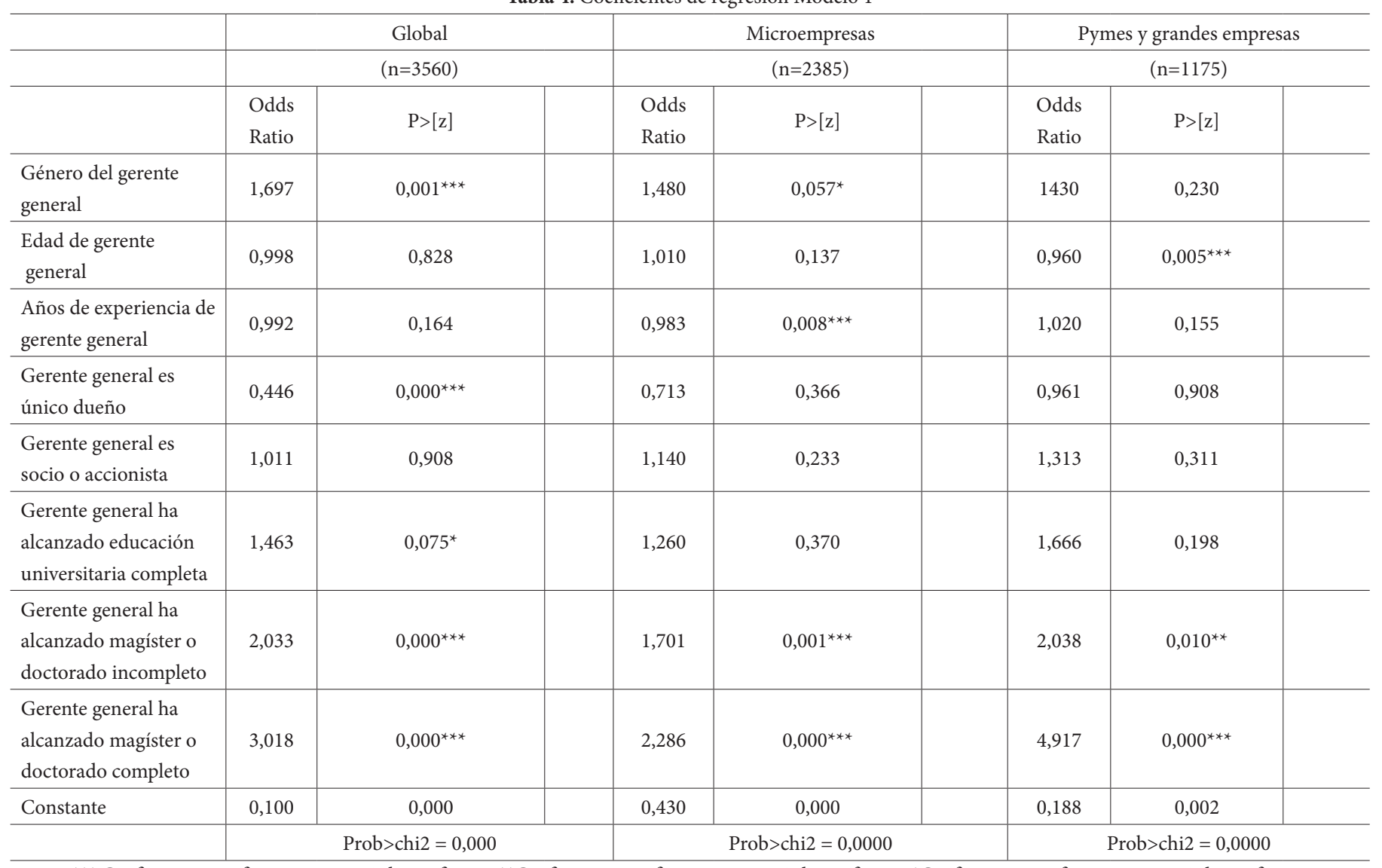

${ }^{* * *}$ Coeficiente significativo con $99 \%$ de confianza, ${ }^{* *}$ Coeficiente significativo con $95 \%$ de confianza, ${ }^{*}$ Coeficiente significativa con $90 \%$ de confianza.

Tabla 5. Probabilidad incremental de variables con coeficientes significativos

\begin{tabular}{l|c|l}
\hline & Odds Ratio & \multicolumn{1}{|c}{ Probabilidad de realizar investigación y desarrollo } \\
\hline Gerente general de género masculino & 1,697 & Probabilidad mayor en $62,97 \%$ si gerente general es de género masculino \\
\hline Gerente general es único dueño de la empresa & 0,998 & Probabilidad mayor en $49,94 \%$ si gerente general no es único dueño \\
\hline $\begin{array}{l}\text { Gerenta general ha alcanzado magíster o doctorado } \\
\text { incompleto }\end{array}$ & 2,033 & $\begin{array}{l}\text { Probabilidad mayor en } 67,02 \% \text { si gerente general ha alcanzado magíster o doctorado } \\
\text { incompleto }\end{array}$ \\
\hline $\begin{array}{l}\text { Gerenta general ha alcanzado magíster o doctorado } \\
\text { completo }\end{array}$ & 3,018 & $\begin{array}{l}\text { Probabilidad mayor en } 75,11 \% \text { si gerente general ha alcanzado magíster o doctorado } \\
\text { completo }\end{array}$ \\
\hline
\end{tabular}

Al dividir las encuestas por tamaño de empresas, se aprecia que la diferencia de género sólo es significativa en el grupo de microempresas, es decir, la probabilidad de realizar actividades de investigación y desarrollo en una empresa con gerente general con género hombre es mayor sólo para este estrato. También en el grupo de microempresas, se aprecia que a mayor cantidad de años de experiencia del gerente general, menor el probabilidad de que la empresa realice actividades de investigación y desarrollo, y que al alcanzar educación de magíster y doctorado completa e incompleta, incrementa la probabilidad de que la empresa efectúe actividades I+D.

Para el caso de las grandes empresas, los coeficientes significativos son similares, sin embargo, la edad del gerente general sustituye a los años de experiencia como variable con coeficiente significativo.

\section{Modelo 2}

El Modelo 2 de regresión logística, muestra los resultados de análisis de 3560 encuestas divididos por industria y tamaño de empresas. Los resultados son presentados en Tabla 6 .

En la industria manufacturera sólo los años de experiencia del gerente general, en el estrato de microempresas, muestra relación negativa con actividades $\mathrm{I}+\mathrm{D}$ con coeficiente significativo.

En la industria de comercio al por mayor y al por menor; reparación de vehículos automotores, motocicletas, efectos personales y enseres domésticos, dentro del estrato de microempresas, mayor experiencia del gerente general se relaciona con menor actividad $\mathrm{I}+\mathrm{D}$, también, 
alcanzar niveles de educación o magíster incompletos y completos incrementa la probabilidad de realizar investigación y desarrollo en la empresa. En el estrato pymes y grandes empresas de esta industria, el género masculino del gerente general se relaciona en forma positiva con participación en $\mathrm{I}+\mathrm{D}$, la edad del gerente general presenta relación negativa, y los estudios de magíster o doctorado completos e incompletos relación positiva.
La industria Actividades inmobiliarias empresariales y de alquiler refleja coeficientes positivos sólo en el estrato de pymes y grandes empresas. En este grupo, la edad del gerente presenta relación negativa con $\mathrm{I}+\mathrm{D}$, y los años de experiencia del gerente general relación positiva. Adicionalmente, la condición gerente general es dueño o accionista presenta relación positiva con $\mathrm{I}+\mathrm{D}$, y alcanzar educación de magíster o doctorado completo relación positiva.

Tabla 6. Coeficientes de regresión Modelo 2

\begin{tabular}{|c|c|c|c|c|c|c|c|c|c|c|c|c|}
\hline & \multicolumn{4}{|c|}{ Industrias Manufactureras } & \multicolumn{4}{|c|}{$\begin{array}{l}\text { Comercio al por mayor y al por menor; repa- } \\
\text { ración de vehículos automotores, motocicle- } \\
\text { tas, efectos personales y enseres domésticos }\end{array}$} & \multicolumn{4}{|c|}{$\begin{array}{c}\text { Actividades Inmobiliarias, Empresariales } \\
\text { y de Alquiler }\end{array}$} \\
\hline & \multicolumn{2}{|c|}{$\begin{array}{l}\text { Microempresas } \\
\qquad(\mathrm{n}=707)\end{array}$} & \multicolumn{2}{|c|}{$\begin{array}{l}\text { Pymes y grandes } \\
\qquad(\mathrm{n}=324)\end{array}$} & \multicolumn{2}{|c|}{$\begin{array}{l}\text { Microempresas } \\
\quad(\mathrm{n}=1001)\end{array}$} & \multicolumn{2}{|c|}{$\begin{array}{l}\text { Pymes y grandes } \\
\quad(\mathrm{n}=438)\end{array}$} & \multicolumn{2}{|c|}{$\begin{array}{l}\text { Microempresas } \\
\qquad(\mathrm{n}=677)\end{array}$} & \multicolumn{2}{|c|}{$\begin{array}{l}\text { Pymes y grandes } \\
\quad(\mathrm{n}=413)\end{array}$} \\
\hline & $\begin{array}{l}\text { Odds } \\
\text { Ratio }\end{array}$ & $\mathrm{P}>[\mathrm{z}]$ & $\begin{array}{l}\text { Odds } \\
\text { Ratio }\end{array}$ & $\mathrm{P}>[\mathrm{z}]$ & $\begin{array}{l}\text { Odds } \\
\text { Ratio }\end{array}$ & $\mathrm{P}>[\mathrm{z}]$ & $\begin{array}{l}\text { Odds } \\
\text { Ratio }\end{array}$ & $\mathrm{P}>[\mathrm{z}]$ & $\begin{array}{l}\text { Odds } \\
\text { Ratio }\end{array}$ & $\mathrm{P}>[\mathrm{z}]$ & $\begin{array}{l}\text { Odds } \\
\text { Ratio }\end{array}$ & $\mathrm{P}>[\mathrm{z}]$ \\
\hline $\begin{array}{l}\text { Género del gerente } \\
\text { general }\end{array}$ & 1,033 & 0,93 & 2,024 & 0,220 & 1,965 & 0,100 & 3,038 & $0,094^{*}$ & 1,257 & 0,537 & 0,773 & 0,557 \\
\hline $\begin{array}{l}\text { Edad de gerente } \\
\text { general }\end{array}$ & 1,012 & 0,28 & 0,995 & 0,815 & 1,004 & 0,785 & 0,953 & $0,096^{*}$ & 1,002 & 0,873 & 0,919 & $0,004^{* * *}$ \\
\hline $\begin{array}{l}\text { Años de experiencia } \\
\text { de gerente general }\end{array}$ & 0,976 & $0,012^{* *}$ & 0,991 & 0,640 & 0,977 & $0,058^{*}$ & 1,010 & 0,739 & 1,005 & 0,636 & 1,063 & $0,028^{* *}$ \\
\hline $\begin{array}{l}\text { Gerente general es } \\
\text { único dueño }\end{array}$ & 0,240 & 0,182 & 0,518 & 0,204 & 1,473 & 0,457 & 1,676 & 0,456 & 2,071 & 0,317 & 1,114 & 0,867 \\
\hline $\begin{array}{l}\text { Gerente general es } \\
\text { socio o accionista }\end{array}$ & 1,184 & 0,361 & 0,524 & 0,152 & 1,279 & 0,240 & 1,510 & 0,523 & 1,391 & 0,100 & 2,087 & $0,083^{*}$ \\
\hline $\begin{array}{l}\text { Gerente general ha } \\
\text { alcanzado educación } \\
\text { universitaria completa }\end{array}$ & 0,723 & 0,436 & 1,453 & 0,515 & 1,582 & 0,342 & 3,186 & 0,115 & 1,713 & 0,296 & 1,210 & 0,832 \\
\hline $\begin{array}{l}\text { Gerente general ha } \\
\text { alcanzado magíster o } \\
\text { doctorado incompleto }\end{array}$ & 1,153 & 0,561 & 1,470 & 0,374 & 2,587 & $0,002^{* * *}$ & 3,307 & $0,04^{* *}$ & 1,125 & 0,719 & 2,554 & 0,100 \\
\hline $\begin{array}{l}\text { Gerente general ha } \\
\text { alcanzado magíster o } \\
\text { doctorado completo }\end{array}$ & 1,483 & 0,178 & 2,345 & 0,337 & 4,211 & $0^{* * *}$ & 2,962 & 0,364 & 1,622 & 0,167 & 8,410 & $0,001^{* * *}$ \\
\hline \multirow[t]{2}{*}{ Constante } & 0,421 & 0,153 & $-0,143$ & 0,074 & 0,430 & $0^{* * *}$ & 0,731 & $0,046^{* *}$ & 0,126 & 0,002 & 0,592 & 0,640 \\
\hline & \multicolumn{2}{|c|}{ Prob $>$ chi $2=0,0319$} & \multicolumn{2}{|c|}{$\begin{array}{c}\text { Prob }>\text { chi } 2= \\
0,3938\end{array}$} & \multicolumn{2}{|c|}{ Prob $>$ chi $2=0,0000$} & \multicolumn{2}{|c|}{ Prob $>$ chi $2=0,0850$} & \multicolumn{2}{|c|}{ Prob $>$ chi $2=0,3897$} & \multicolumn{2}{|c|}{ Prob $>$ chi $2=0,0001$} \\
\hline
\end{tabular}

${ }^{* *}$ Coeficiente significativo con $99 \%$ de confianza, ${ }^{* *}$ Coeficiente significativo con $95 \%$ de confianza, ${ }^{*}$ Coeficiente significativa con $90 \%$ de confianza.

Para comprobar la ausencia de multicolinealidad en las variables independientes, se ha realizado Análisis de Tolerancia (TOL) y de Factor de Inflación de la Varianza (VIF). En este análisis se obtienen valores TOL cercanos a 1 y de VIF menores a 2 con promedio 1,35 . No se encuentra evidencia de alta multicolinealidad entre las variables independientes.

\section{Conclusiones}

La presente investigación busca estudiar la relación de: participación en propiedad, nivel educacional, edad, años de experiencia profesional y género del gerente general, con actividades de investigación y desarrollo en empresas en Chile. Los hallazgos obtenidos, muestran que los resultados de investigaciones previas se replican parcialmente en empresas en Chile, según tamaño de empresa y tipo de industria.

Se evidencia que un mayor nivel educacional alcanzado por el gerente general se asocia con mayor propensión a realizar investigación y desarrollo, considerando algunas industrias y tamaños de empresas, esta idea es respaldada por Lin el al. (2011). También, que empresas con gerentes generales que son únicos dueños, presentan menor probabilidad de realizar investigación y desarrollo, premisa coherente con lo planteado por Hirshleifer y Thakor, (1992) y Massis, Frattini, y Lichtenthaler, (2013). Adicionalmente, se observa como tendencia, 
que la experiencia y edad del gerente incide en forma negativa en participación de empresas en $\mathrm{I}+\mathrm{D}$, resultado consistente con lo planteado por Marshall et al. (2006), Por último, que existe relación entre el género masculino y una mayor probabilidad de realizar investigación y desarrollo en la empresa, resultado coherente premisas de Huang y Kisgen (2013), y Faccio et al. (2016).

Los resultados obtenidos muestran que la condición de alcanzar estudios de magíster o doctorado completos, al considerar la muestra global, presenta la mayor incidencia sobre la variable dependiente, incrementando la probabilidad de realizar I+D en $75,11 \%$. Según este resultado, incrementar la formación de postgrados en los gerentes generales impactaría positivamente la participación de empresas en actividades de investigación y desarrollo, y con ello a contribuir a la competitividad de empresas.

Desde la perspectiva de asignación de recursos públicos, la evidencia obtenida facilita el proceso de diagnóstico y evaluación de empresas postulantes en programas de apoyo a la investigación y desarrollo. La propensión hacia la innovación y las capacidades de $\mathrm{I}+\mathrm{D}$, pueden ser estimadas a través de las características del gerente general, por tanto, algunas de estas condiciones, por ejemplo, la formación del gerente general, son pertinentes de evaluación en el proceso de adjudicación de fondos, como los otorgados por la Corporación de Fomento y la Producción de Chile a través de concursos FICr o FDNr. Adicionalmente, las empresas dirigidas por gerentes generales con condiciones relacionadas en forma negativa con $\mathrm{I}+\mathrm{D}$ pueden ser objeto de programas especiales financiados con fondos nacionales o regionales.

Bajo la visión de dirección de empresas, los resultados aportan a la selección de gerentes generales con condiciones facilitadoras para investigación y desarrollo, también para el desarrollo de programas de formación gerencial, de incentivos financieros para el gerente y de financiamiento de postgrados.

Respecto de la evidencia que relaciona experiencia del gerente general con menor participación de empresas en I + D, es pertinente implementar incentivos para la incorporación de directivos con menor experiencia en las empresas, y desarrollar convenios entre universidades y empresas para facilitar la incorporación de egresados en cargos de alta dirección.

La relación negativa entre participación en propiedad y participación $\mathrm{I}+\mathrm{D}$ de las empresas, puede ser abordada a través de entrega de subsidios para $\mathrm{I}+\mathrm{D}$, que prioricen a empresas con alta concentración de propiedad, o por medio de programas de formación que busquen fomentar beneficios de I+D en empresas concentradas, y que presten apoyo en su implementación.

Para la relación negativa entre género femenino y participación en I $+\mathrm{D}$ en la empresa, se propone el desarrollo de programas de formación y difusión orientado a gerentes de género femenino y la aplicación de subsidios a la innovación dirigido a empresas dirigidas por estas gerentes.
Las propuestas descritas, apoyarían la participación de organizaciones en investigación y desarrollo, y con ello, a la competitividad y crecimiento de empresas, según lo planteado por (O’Brien, 2003). Adicionalmente, las propuestas pueden ser consideradas por países latinoamericanos con economías emergentes, como Brasil, México y Colombia.

Se reconocen como limitaciones del estudio la inclusión de sólo 3 industrias, también, que las pymes y grandes empresas son estudiadas en conjunto, y que la edad de la empresa, variable con relación inversa a actividades I+D (Hansen 1992), no ha sido utilizada como variable de control. Adicionalmente, para evaluar actividades de investigación y desarrollo, sólo se incluye una pregunta en la encuesta, sin incorporar monto de inversión, número de patentes, número de productos diseñados o cantidad de publicaciones realizas, variables recomendada por Brown y Svenson (1998) para medición de outputs de I+D. Por último, la formación del gerente general no es estudiada por área disciplina, factor que puede ser relevante, pues podrían ser reconocidas disciplinas asociadas con mayor propensión a I+D en las empresas. A pesar de estas limitaciones, la cantidad de empresas incluidas en la muestra permite representar la participación de organizaciones con características diversas.

Futuras investigaciones pueden incorporar la variable edad de la empresa, mayor número de industrias y reconocer diferencias en organizaciones dirigidas por gerentes con formación en diferentes áreas de especialización. Además, utilizar nuevos indicadores para medir participación de empresas en actividades I+D.

\section{Acerca de los Autores:}

Gustavo Barrera Verdugo es Magíster en Marketing de la Universidad de Chile y Doctorando en Ciencias de la Administración en Universidad de Santiago de Chile. Actualmente se desempeña como Profesor de Postgrado en la Universidad Tecnológica de Chile. Sus intereses en relación con investigación se vinculan con perfil de gerencia y de consumidores. Imparte docencia en marketing, innovación y estrategia de empresa.

Eugenio Bisama Castillo es Doctor en Economía y Dirección de Empresas por la Universidad de Deusto, España. Actualmente se desempeña como Profesor de Postgrado en la Universidad Tecnológica de Chile. Sus intereses en relación con investigación se vinculan con valor compartido y stakeholders. Imparte docencia en estrategia de empresa, modelos de negocio e innovación.

\section{Referencias}

Barker, V. L., Mueller, G. C. (2002). CEO characteristic and firm R\&D spending. Management Science 48 (1): 782-801. http://dx.doi. org/10.1287/mnsc.48.6.782.187

Barua, A., Davidson, L., Rama, D., \& Thiruvadi, S. (2010). CFO gender and accruals quality. Accounting Horizons, 24(1), 25-39. http:// dx.doi.org/10.2308/acch.2010.24.1.25 
Bromiley, P., Rau, D., \& Zhang, Y. (2016). Is R\&D risky? Strategic Management Journal. http://dx.doi.org/10.1002/smj.2520

Brown, M. G., \& Svenson, R. A. (1998). RTM Classic: Measuring R\&D Productivity. Research-Technology Management, 41(6), 30-35.

Block, J. H. (2012). R\&D investments in family and founder firms: An agency perspective. Journal of Business Venturing, 27(2), 248-265. http://dx.doi.org/10.1016/j.jbusvent.2010.09.003

Cazier, R. A. (2011). Measuring R\&D curtailment among short-horizon CEOs. Journal of Corporate Finance, 17(3), 584-594. http:// dx.doi.org/10.1016/j.jcorpfin.2011.02.005

Chen, H. L, Hsu, W. T. (2009). Family ownership, board independence and R\&D investment. Family Business Review 22 (4): 347-362. http://dx.doi.org/10.1177/0894486509341062

Chen, S., Lin, B., Lu, R., \& Ma, H. (2016). Pay for accounting performance and R\&D investment: Evidence from China. Internatio- nal Review of Economics \& Finance, 44, 142-153. http://dx.doi. org/10.1016/j.iref.2016.04.004

Ettlie, J. E. (1998). R\&D and global manufacturing performance. Management Science, 44(1), 1-11. http://dx.doi.org/10.1287/mnsc.44.1.1

Faccio, M., Marchica, M. T., \& Mura, R. (2016). CEO gender, corporate risk-taking, and the efficiency of capital allocation. Available at SSRN 2021136. http://dx.doi.org/10.1016/j.jcorpfin.2016.02.008

Farag, H., \& Mallin, C. (2016). The influence of CEO demographic characteristics on corporate risk-taking: evidence from Chinese IPOs. The European Journal of Finance, 1-30. http://dx.doi.org/10.10 80/1351847x.2016.1151454

Ghosh, A., Moon, D., Tandon, K. (2007). CEO ownership and discretionary investments. Journal of Business Finance \& Accounting 34 (5-6): 819-839. http://dx.doi.org/10.1111/j.1468-5957.2007.02011.x

Hambrick, D. C., Mason, P. A. (1984). Upper echelons: The organization as a reflection of its top managers. Academy of Management Review 9 (2): 193-206. http://dx.doi.org/10.5465/amr.1984.4277628 Hansen, J. A. (1992). Innovation, firm size, and firm age. Small Business Economics, 4(1), 37-44. http://dx.doi.org/10.1007/BF00402214

Hirshleifer, D., \& Thakor, A. V. (1992). Managerial conservatism, project choice, and debt. Review of Financial Studies, 5(3), 437-470. https://doi.org/10.1093/rfs/5.3.437

Huang, J., \& Kisgen, D. (2013). Gender and corporate finance: Are male executives overconfident relative to female executives? Jour- nal of Financial Economics, 108, 822-839. https://doi.org/10.2139/ ssrn.1263990

Hsu, C. W., Lien, Y. C., \& Chen, H. (2015). R\&D internationalization and innovation performance. International Business Review, 24(2), 187-195. https://doi.org/10.1016/j.ibusrev.2014.07.007
Jensen, M. C. (1993). The modern industrial revolution, exit and the failure of internal control systems. Journal of Finance 48 (3): 831-881. https://doi.org/10.2307/2329018

Kor, Y. Y. (2006). Direct and interaction effects of top management team and board compositions on R\&D investment strategy. Strategic Management Journal, 27(11), 1081-1099. https://doi.org/10.1002/ smj.554

Lee, W. S., \& Moon, J. (2016). Determinants of CEO strategic risktaking in the airline industry. Tourism Management Perspectives, 18, 111-117. https://doi.org/10.1016/j.tmp.2016.01.009

Lin, C., Lin, P., Song, F. M., \& Li, C. (2011). Managerial incentives, CEO characteristics and corporate innovation in China's private sector. Journal of Comparative Economics, 39(2), 176-190. https://doi. org/10.1016/j.jce.2009.12.001

López-Sánchez, J. I., Minguela-Rata, B., Rodriguez-Duarte, A., Sandulli, F.D. (2006). R\&D resources and diversification: controlling for indirect diversification and endogeneity. International Journal of Technology Management 35 (1-4): 136-155. https://doi.org/10.1504/ ijtm.2006.009232

Matta, E., Beamish, P. W. (2008). The accentuated CEO career horizon problem: Evidence from international acquisitions. Strategic Management Journal 29 (7): 683-700. https://doi.org/10.1002/smj.680

De Massis, A., Frattini, F., \& Lichtenthaler, U. (2012). Research on technological innovation in family firms: Present debates and future directions. Family Business Review, 0894486512466258. https://doi. org/10.1177/0894486512466258

Marshall, J. P., Sorenson, R., Brigham, K., Wieling, E., Reifman, A., \& Wampler, R. S. (2006). The paradox for the family firm CEO: Owner age relationship to succession-related processes and plans. Journal of Business Venturing, 21(3), 348-368. https://doi.org/10.1016/j.jbusvent.2005.06.004

Minnick, K., \& Noga, T. (2010). Do corporate governance characteristics influence tax management? Journal of corporate finance, 16(5), 703-718. https://doi.org/10.1016/j.jcorpfin.2010.08.005

Ministerio de Economía, Fomento y Turismo de Chile (2015). Tercera Encuesta Longitudinal de Empresas (ELE3) 2015. [En línea] $<$ http:// www.economia.gob.cl/estudios-y-encuestas/encuestas/encuestas-de- emprendimiento-y-empresas/tercera-encuesta-longitudinal-de-em- presas>. [Consulta: Enero 2016]

Morgan Stanley Capital International (2016), MSCI World Index Stock 2016. [En línea] <https://www.msci.com/world>. [Consulta: Octubre 2016]

Organización para la Cooperación y Desarrollo Económicos (2015). Medición de las Actividades Científicas y Tecnológicas, documento conocido como Manual de Frascati. Cap. 2; 2.1 
O'brien, J. P. (2003). The capital structure implications of pursuing a strategy of innovation. Strategic Management Journal, 24(5), 415431. https://doi.org/10.1002/smj.308

Serfling, M. A. (2014). CEO age and the riskiness of corporate policies. Journal of Corporate Finance, 25, 251-273. https://doi.org/10.1016/j. jcorpfin.2013.12.013

KS, S., \& Mukherjee, B. (2004). How Much Does R\&D Decision Depend on Firm, Industry, Group and its Interactions?. Industry, Group and Its Interactions. https://doi.org/10.2139/ssrn.645525
Ting, H. I. (2013). CEO turnover and shareholder wealth: Evidence from CEO power in Taiwan. Journal of Business Research, 66(12), 2466-2472. https://doi.org/10.1016/j.jbusres.2013.05.036

Wu, S., Levitas, E., \& Priem, R. L. (2005). CEO tenure and company invention under differing levels of technological dynamism. Academy of Management Journal, 48(5), 859-873. https://doi.org/10.5465/ amj.2005.18803927 